\title{
CRITICAL TIMESCALES AND TIME INTERVALS FOR COUPLED LINEAR PROCESSES
}

\author{
MATTHEW J. SIMPSON ${ }^{\bowtie 1}$, ADAM J. ELLERY ${ }^{1}$, SCOTT W. MCCUE ${ }^{1}$ \\ and RUTH E. BAKER ${ }^{2}$
}

(Received 18 October, 2012; revised 10 January, 2013; first published online 22 April, 2013)

\begin{abstract}
In 1991, McNabb introduced the concept of mean action time (MAT) as a finite measure of the time required for a diffusive process to effectively reach steady state. Although this concept was initially adopted by others within the Australian and New Zealand applied mathematics community, it appears to have had little use outside this region until very recently, when in 2010 Berezhkovskii and co-workers [A. M. Berezhkovskii, C. Sample and S. Y. Shvartsman, "How long does it take to establish a morphogen gradient?" Biophys. J. 99 (2010) L59-L61] rediscovered the concept of MAT in their study of morphogen gradient formation. All previous work in this area has been limited to studying single-species differential equations, such as the linear advection-diffusionreaction equation. Here we generalize the concept of MAT by showing how the theory can be applied to coupled linear processes. We begin by studying coupled ordinary differential equations and extend our approach to coupled partial differential equations. Our new results have broad applications, for example the analysis of models describing coupled chemical decay and cell differentiation processes.
\end{abstract}

2010 Mathematics subject classification: primary 35A09.

Keywords and phrases: reaction-diffusion equation, coupled reaction-diffusion equations, steady state, critical time.

\section{Introduction}

Estimating the time required for an advection-diffusion-reaction process to effectively reach steady state is important in many applications [8]. Such an estimate is usually referred to as a critical time [12-14]. In 1991, McNabb [21, 22] introduced the theory of mean action time (MAT) as a critical time for a diffusive process related to an industrial heat transfer problem. McNabb's analysis showed that the MAT could be determined without solving the partial differential equation (PDE) for the transient

\footnotetext{
${ }^{1}$ Mathematical Sciences, Queensland University of Technology, Brisbane 4001, Australia; e-mail: matthew.simpson@qut.edu.au, a.ellery@qut.edu.au, scott.mccue@qut.edu.au.

${ }^{2}$ Centre for Mathematical Biology, Mathematical Institute, University of Oxford, 24-29 St Giles', Oxford OX1 3LB, UK; e-mail: ruth.baker@maths.ox.ac.uk.

(C) Australian Mathematical Society 2013, Serial-fee code 1446-1811/2013 \$16.00
} 
solution [21, 22]. Although McNabb's results were adopted within the Australian and New Zealand applied mathematics community [17, 18], there seems to have been little interest in this fundamental concept beyond Australia and New Zealand until very recently when Berezhkovskii and co-workers introduced a critical time that they called the local accumulation time (LAT) [2-4, 11, 15]. Berezhkovskii and co-workers studied a one-dimensional reaction-diffusion PDE as a model of morphogen gradient formation. For a particular initial condition, $C_{0}(x)$, they found the time-dependent solution, $C(x, t)$, the steady state solution $C_{\infty}(x)=\lim _{t \rightarrow \infty} C(x, t)$, and the LAT [2$4,11,15]$. In a recent paper [8] we showed that Berezhkovskii's definition of LAT is equivalent to McNabb's definition of MAT. Furthermore, we considered the MAT for a general one-dimensional advection-diffusion-reaction PDE and found that the moments of the associated probability density function can be evaluated to give the variance of action time (VAT), skew of action time (SAT), kurtosis of action time (KAT) and so on [9].

All previous analyses of MAT have focused on a single linear differential equation [2-4, 8, 9, 11, 15, 21, 22]. Here, we extend McNabb's definition of MAT so that we can find the critical time of coupled linear differential equations. We begin our analysis by studying coupled ordinary differential equations (ODEs) and subsequently show how the results can be applied to coupled PDEs.

\section{Coupled ordinary differential equations}

Applications of linear coupled ODEs include the study of sequential first-order reaction networks that are used to represent chemical and biochemical processes including radioactive decay [27], nitrification-denitrification processes [5, 19] and biodegradation of chlorinated solvents $[6,28,29]$. Here, we show how to apply the concept of MAT to linear coupled ODEs by considering a model of the biodegradation of a common chlorinated solvent, called tetrachloroethene (PCE), which decays sequentially into trichloroethene (TCE), dichloroethylene (DCE) and vinyl chloride (VC) [6, 28, 29]. PCE and TCE are commonly used industrial solvents, such as dry cleaning fluids, and are the most frequent contaminants found in groundwater supplies [28]. Although PCE and TCE are recognized as carcinogens [20], their biotransformation into $\mathrm{VC}$ is of particular concern as $\mathrm{VC}$ is highly toxic and considered to be more of a biological hazard than the parent species [20].

A typical model of PCE decay in a well-stirred batch reactor can be written as

$$
\begin{aligned}
& \frac{d C_{1}}{d t}=-k_{1} C_{1}, \\
& \frac{d C_{2}}{d t}=k_{1} C_{1}-k_{2} C_{2}, \\
& \frac{d C_{3}}{d t}=k_{2} C_{2}-k_{3} C_{3}, \\
& \frac{d C_{4}}{d t}=k_{3} C_{3}-k_{4} C_{4},
\end{aligned}
$$


where $C_{1}, C_{2}, C_{3}$ and $C_{4}$ are the molar concentrations of PCE, TCE, DCE and $\mathrm{VC}$, respectively, and $k_{i}>0$ is the first-order decay rate of the $i$ th species for $i=$ $1,2,3,4[6,29]$. This model illustrates the importance of the coupled nature of the decay process. Typically, the initial conditions used to model the decay of chlorinated solvent waste are $C_{1}(0) \geq 0$ and $C_{2}(0) \geq 0$, since PCE and TCE are often present in disposed solvent waste, while the initial conditions for DCE and VC are taken to be $C_{3}(0)=0$ and $C_{4}(0)=0$, as these species are initially absent $[6,28]$. The system of ODEs governing this decay process, given by equations (2.1)-(2.4), has the properties that $C_{4}(t)>0$ for all $t>0$, and $C_{4}(t) \rightarrow 0$ as $t \rightarrow \infty$. In other words, the solution of the mathematical model predicts that VC forms instantaneously and remains present for an infinite amount of time, although it decays to zero in the long-time limit. This sort of result is not very helpful from a practical perspective, however, as it is not immediately clear how we can make quantitative conclusions about the time lag required for significant quantities of $\mathrm{VC}$ to form, and the timescale over which $\mathrm{VC}$ is persistent in the system before its concentration is effectively negligible. This issue is particularly relevant for the PCE decay system, given that VC is highly toxic [20].

If, instead of dealing with a multispecies first-order reaction network, we are dealing with a single species system, given by

$$
\frac{d C_{1}}{d t}=-k_{1} C_{1}
$$

then it is clear that the important timescale is simply $t=1 / k_{1}$ [22] since there is only one inherent timescale present in equation (2.5) [1]. However, for multispecies systems with $N>1$ species, such as (2.1)-(2.4) with $N=4$, we have multiple timescales inherent in the mathematical model, $t_{i}=1 / k_{i}$ for $i=1,2, \ldots, N$, and it is not obvious how these competing timescales govern the overall dynamics of each species [12-14]. This difficulty is further complicated by the fact that the relevant features of the system will also depend on the initial conditions $C_{i}(0)$, for $i=1,2, \ldots, N$.

The exact solution of equations (2.1)-(2.4) can be found using any symbolic package such as Maple or Mathematica. The exact solution is very lengthy and we do not present the details here. Furthermore, we contend that such exact solutions are not always insightful for two reasons. First, the exact solution for such coupled ODEs is straightforward to evaluate and interpret only when the number of species is relatively small, say $N<4$. Second, the details of the exact solution depend on the decay rates, $k_{i}$ for $i=1,2, \ldots, N$. For example, the exact solution for (2.1)-(2.4) can be written as a linear combination of exponential complementary functions $\mathrm{e}^{-k_{i} t}$ for $i=1,2,3,4$, which is relevant for the case in which the reaction rates are distinct. Otherwise, in the case of two or more equal decay rates, some of the complementary functions will be products of polynomials and exponentials, and thus the form of the exact solution depends upon whether the reaction rates are distinct or repeated. Of course, it is always possible to solve equations (2.1)-(2.4) numerically, and such numerical solutions have the advantage that they do not depend on whether the reaction rates are distinct or repeated. However, taking a purely numerical approach provides 
no analytical insight into how the overall timescales depend on the various decay rates, $k_{i}$, and initial concentrations, $C_{i}(0)$, for $i=1,2, \ldots, N$.

Here, we present an alternative approach for analysing sequential first-order reaction networks that avoids both these limitations. Instead of analysing the solution, $C_{i}(t)$, for $i=1,2, \ldots, N$, we take an averaged approach and determine the MAT and VAT for each species. As mentioned in Section 1, all previous applications of MAT dealt with a single-species process only, and our approach is the first attempt to apply these concepts to coupled problems.

2.1. Analysis We consider a general sequential first-order reaction network:

$$
\begin{aligned}
& \frac{d C_{1}}{d t}=-k_{1} C_{1}, \\
& \frac{d C_{i}}{d t}=k_{i-1} C_{i-1}-k_{i} C_{i}, \quad \text { for } i=2,3, \ldots, N,
\end{aligned}
$$

where $k_{i}>0$ for $i=1,2, \ldots, N$.

To make progress within the MAT and VAT framework [9], we introduce a new variable $S_{n}(t)=\sum_{i=1}^{n} C_{i}(t)$, and we note that for all practical chemical decay problems we have $S_{i}(0)>0$ for $i=1,2, \ldots, N$. Under these conditions, the quantity

$$
F_{n}(t)=1-\frac{S_{n}(t)}{S_{n}(0)}
$$

acts as a cumulative distribution function since $F_{n}(0)=0$ and $F_{n}(t) \rightarrow 1^{-}$as $t \rightarrow \infty$ for each $n=1,2, \ldots, N$. The associated probability density function can be written as

$$
f_{n}(t)=-\frac{1}{S_{n}(0)} \frac{d S_{n}(t)}{d t} .
$$

Our use of the $S_{n}(t)$ variable is essential if we are going to interpret equation (2.6) as a cumulative distribution function. For the simple case where $i=1$, where we have $d C_{1} / d t=-k_{1} C_{1}$, it is straightforward to interpret the quantity $F(t)=1-C_{1}(t) / C_{1}(0)$ as a cumulative distribution function, and the quantity $f(t)=d F(t) / d t$ as a probability density function, of which we can evaluate the associated moments [9]. If, on the other hand, we consider a coupled problem with $i=2$, then the quantity $F(t)=$ $1-C_{2}(t) / C_{2}(0)$ cannot be interpreted as a cumulative distribution function since $F(t)=1-C_{2}(t) / C_{2}(0)$ is not a monotonically increasing function of $t$.

We can calculate the moments of (2.7), including the MAT, which is given by

$$
T_{n}=\int_{0}^{\infty} t f_{n}(t) d t,
$$

and the VAT,

$$
V_{n}=\int_{0}^{\infty}\left(t-T_{n}\right)^{2} f_{n}(t) d t
$$


Higher-order moments can be defined in an obvious way [9]; however, we do not consider these here.

To calculate the MAT and VAT, we first evaluate the integrals

$$
\int_{0}^{\infty} t \frac{d S_{n}(t)}{d t} d t=-\sum_{i=1}^{n} \frac{S_{i}(0)}{k_{i}}, \quad \int_{0}^{\infty} t^{2} \frac{d S_{n}(t)}{d t} d t=-\sum_{i=1}^{n} \frac{2}{k_{i}}\left[\sum_{j=1}^{i} \frac{S_{j}(0)}{k_{j}}\right],
$$

where we have made use of the fact that $S_{i}(t) \rightarrow 0$ exponentially fast as $t \rightarrow \infty$ for $i=1,2, \ldots, N[8,9]$. The details regarding how to evaluate the integrals in equation (2.8) are given in Appendix A. Using (2.8), we find that the MAT is

$$
T_{n}=\frac{1}{S_{n}(0)} \sum_{i=1}^{n} \frac{S_{i}(0)}{k_{i}}
$$

and the VAT is

$$
V_{n}=\frac{1}{S_{n}(0)} \sum_{i=1}^{n}\left[\frac{2}{k_{i}} \sum_{j=1}^{i} \frac{S_{j}(0)}{k_{j}}\right]-\frac{2 T_{n}}{S_{n}(0)} \sum_{i=1}^{n} \frac{S_{i}(0)}{k_{i}}+T_{n}^{2} .
$$

In the simple case where $n=1$, equation (2.9) gives $T_{1}=1 / k_{1}$ which coincides with the results of McNabb [21, 22], as expected.

For a coupled problem with $N>1$ species, we see that (2.9) gives us a very simple expression showing how the mean timescales depend on $k_{i}$ and $C_{i}(0)$, for $i=1,2, \ldots, N$. One of the great advantages of using this approach is that the MAT and VAT for each species can be evaluated very easily without the need for solving the governing equation for $C_{i}(t)$, for $i=1,2, \ldots, N[8,9,17,22]$. Therefore, our approach avoids the need for working with any numerical or analytical solutions of the governing differential equations, which can be problematic for the reasons highlighted in Section 1.

2.2. Results Figure 1(a)-(d) shows a numerical solution of equations (2.1)-(2.4) with typical reaction rates for the PCE decay system [6]. In each profile we identify the MAT, $T_{i}$, for $i=1,2,3,4$, which we consider to be a critical time for the $i$ th species. In addition, we identify a critical time interval using the VAT, $V_{i}$, for $i=1,2,3$, 4, which we take to be $t \in\left[T_{i}-\sqrt{V_{i}}, T_{i}+\sqrt{V_{i}}\right]$. Profiles in Figure 1 superpose $C_{i}(t), T_{i}$ and the critical time interval for each species $i=1,2,3,4$.

Comparing the numerical solutions of equations (2.1)-(2.4) with the MAT and VAT results in Figure 1 indicates that our results allow us to identify an important time interval for the dynamics of each species. For all values of $t$ prior to the critical time interval, we observe that the concentration of each species is either zero, or relatively small and increasing. Conversely, for all values of $t$ after the critical time interval, the concentration of each species is relatively small and decaying away to zero. This information allows us to make a simple prediction that the critical time interval for the VC profile is $t \in[850,3217]$ days. We emphasize that these critical times and critical 

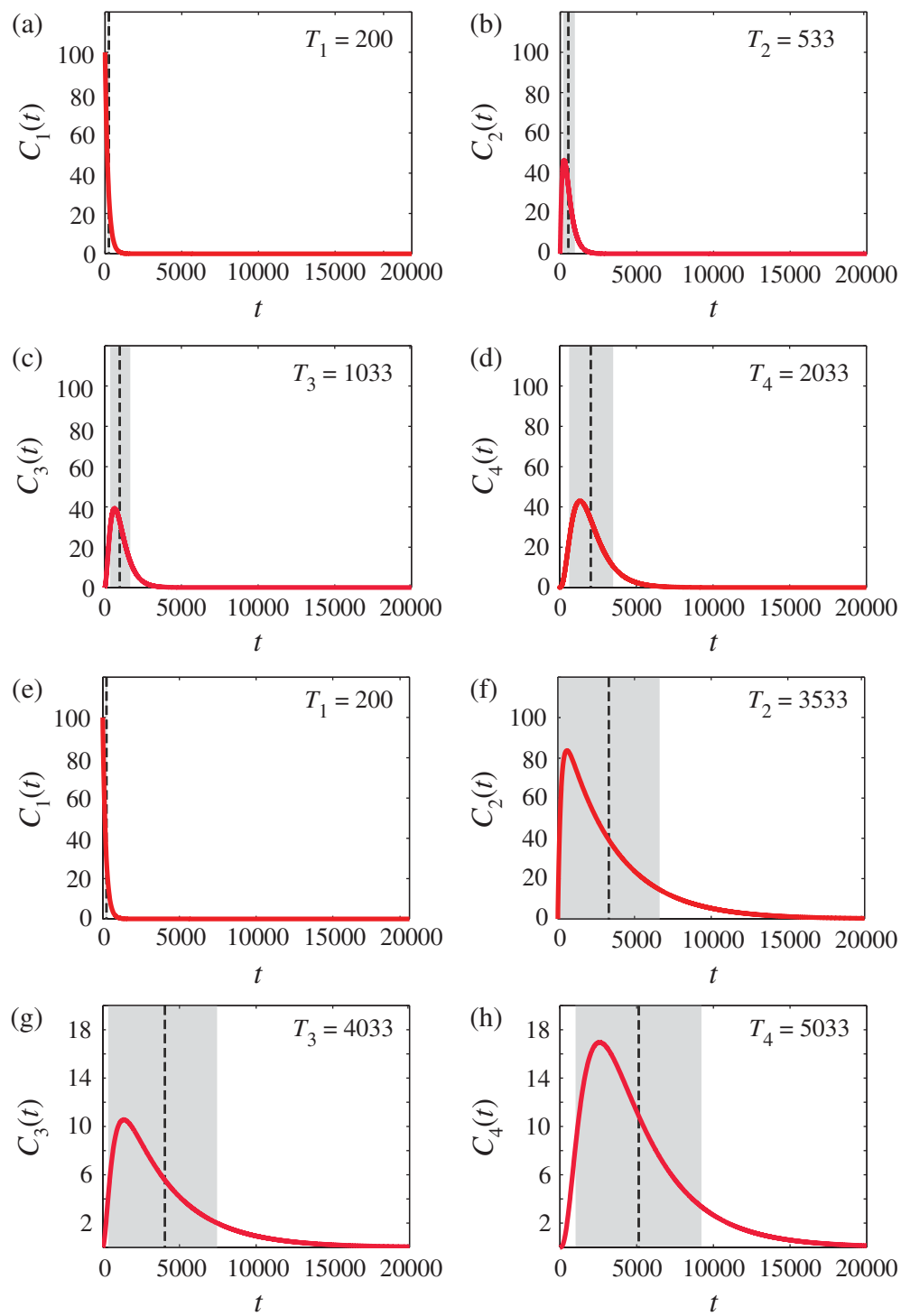

FIGURE 1. Results in (a)-(d) show a numerical solution of (2.1)-(2.4) during the time interval $0 \leq t \leq$ 20000 days, for $C_{1}(0)=100, C_{2}(0)=0, C_{3}(0)=0$ and $C_{4}(0)=0$, with decay rates chosen to reflect PCE, TCE, DCE and VC decay, $k_{1}=0.005, k_{2}=0.003, k_{3}=0.002$ and $k_{4}=0.001$ days $^{-1}$ [6]. Numerical solutions were obtained using a fourth-order Runge-Kutta method [23, 24]. The MAT for each species, $T_{1}=200, T_{2}=533.33, T_{3}=1033.33$ and $T_{4}=2033.33$ days, is shown with a vertical dashed line. We also indicate, by shading, the critical time interval for each species, $\left[T_{i}-\sqrt{V_{i}}, T_{i}+\sqrt{V_{i}}\right]$. Here $V_{1}=200^{2}$, $V_{2}=388.73^{2}, V_{3}=633.33^{2}$ and $V_{4}=1183.70^{2}$ days ${ }^{2}$. Results in (e)-(h) show the same details as (a)-(d), with the same parameters except that the decay rate of TCE is reduced to $k_{2}=0.0003$. Here $T_{1}=200$, $T_{2}=3533.33, T_{3}=4033.33$ and $T_{4}=5033.33$ days, and $V_{1}=200^{2}, V_{2}=3339.30^{2}, V_{3}=3376.60^{2}$ and $V_{4}=3521.50^{2}$ days $^{2}$. 
time intervals have been calculated very simply, for all species, without the need for solving equations (2.1)-(2.4). These results are also very practical since the analytical solution for $C_{4}(t)$, which we do not report here, is a complicated algebraic expression that is not straightforward to interpret. The advantages of this approach are particularly obvious if we are dealing with a reaction network with many more species, say $N=10$, where it would be impractical to generate or interpret an exact analytical solution and repeated numerical simulation of the system of ODEs would not provide any general insight into critical timescales and critical time intervals. Alternatively, our approach provides fast and meaningful insight regardless of the size of the decay chain.

Although the reaction rates for certain sequential first-order reaction networks are known precisely, previous laboratory-scale and field-scale analyses of PCE decay systems have indicated that the first-order decay rates can vary by as much as two orders of magnitude depending on the context of the system [6]. Therefore, it is of interest for us to be able to perform a sensitivity analysis of the critical times and critical timescales for the coupled decay process. Here we show that our approach using MAT and VAT is well suited to conducting such an analysis. We do this by presenting a second set of results in Figure 1(e)-(h). These results are identical to those in Figure 1(a)-(d) except that the TCE decay rate, $k_{2}$, is reduced by an order of magnitude. In response we see that the MATs $T_{2}, T_{3}$ and $T_{4}$ increase. This is consistent with the reduction in $k_{2}$, which should have the effect of delaying the time period in which meaningful concentrations of these species are prevalent. Further, we see that the VATs $V_{2}, V_{3}$ and $V_{4}$ increase, which means that the critical time interval for all downstream species becomes much longer. Our results show that the increase in the critical time and critical time interval is accurately captured by our expressions for MAT and VAT.

\section{Coupled partial differential equations}

The analysis and results provided in Section 2 illustrate that a relatively straightforward change of variable is required before we can evaluate the MAT and VAT for coupled linear ODEs. To generalize our results we show that a similar transformation can be applied to study coupled PDEs. For simplicity we consider the system

$$
\begin{aligned}
& \frac{\partial C_{1}}{\partial t}=D \frac{\partial^{2} C_{1}}{\partial x^{2}}-k_{1} C_{1}, \\
& \frac{\partial C_{2}}{\partial t}=D \frac{\partial^{2} C_{2}}{\partial x^{2}}+k_{1} C_{1}-k_{2} C_{2},
\end{aligned}
$$

where $D>0$ is the diffusivity, $k_{1}$ is the linear decay rate of $C_{1}(x, t)$ and $k_{2}$ is the linear decay rate of $C_{2}(x, t)$. Although we present results for a system with two species, $C_{1}(x, t)$ and $C_{2}(x, t)$, our approach can be extended to systems with $N$ species, $C_{i}(x, t)$ for $i=1,2,3, \ldots, N$.

Various physical processes are encoded in the model system given by equations (3.1)-(3.2). In an obvious extension to the results presented in Section 2, 
equations (3.1)-(3.2) are often used to represent the spatial and temporal distribution of a chemical species $C_{1}(x, t)$, which decays into a second species $C_{2}(x, t)[5,6,19$, 23, 27-29]. In another context, equations (3.1)-(3.2) and variations thereof are used to represent the spatial and temporal distribution of interacting cell populations where one cell type, $C_{1}(x, t)$, differentiates into another cell type, $C_{2}(x, t)[7,16]$.

3.1. Analysis We treat equations (3.1)-(3.2) on the semi-infinite domain $0 \leq x<\infty$ with the trivial initial conditions $C_{1}(x, 0)=0$ and $C_{2}(x, 0)=0$. With boundary conditions $C_{1}(0, t)=1$ and $C_{2}(0, t)=0$, the steady-state solution is

$$
\begin{aligned}
& C_{1 \infty}(x)=\exp \left(-x \alpha_{1}\right), \\
& C_{2 \infty}(x)=\frac{k_{1}}{k_{2}-k_{1}}\left[\exp \left(-x \alpha_{1}\right)-\exp \left(-x \alpha_{2}\right)\right],
\end{aligned}
$$

where $\alpha_{1}=\sqrt{k_{1} / D}, \alpha_{2}=\sqrt{k_{2} / D}$ and $k_{1} \neq k_{2}$. We chose these particular initial conditions and boundary conditions since they have been implemented in previous applications of equations (3.1)-(3.2) [5]. Although we present specific results for this choice of initial conditions and boundary conditions, our framework for calculating the MAT and VAT is general and can be applied to other situations where required [19].

We first derive an expression for the MAT and the VAT for the $C_{1}(x, t)$ component by identifying

$$
F_{1}(t ; x)=1-\frac{C_{1}(x, t)-C_{1 \infty}(x)}{C_{1}(x, 0)-C_{1 \infty}(x)}
$$

as a cumulative distribution function. The associated probability density function is given by

$$
f_{1}(t ; x)=-\frac{\partial}{\partial t}\left[\frac{C_{1}(x, t)-C_{1 \infty}(x)}{C_{1}(x, 0)-C_{1 \infty}(x)}\right] .
$$

With these definitions we can write the MAT as

$$
T_{1}(x)=\int_{0}^{\infty} t f_{1}(t ; x) d t=\frac{1}{g_{1}(x)} \int_{0}^{\infty} t \frac{\partial}{\partial t}\left(C_{1}(x, t)-C_{1 \infty}(x)\right) d t,
$$

where $g_{1}(x)=C_{1 \infty}(x)-C_{1}(x, 0)$. Integrating equation (3.5) by parts and noting that $C_{1}(x, t)-C_{1 \infty}(x)$ decays to zero exponentially fast as $t \rightarrow \infty$ yields

$$
T_{1}(x) g_{1}(x)=\int_{0}^{\infty} C_{1 \infty}(x)-C_{1}(x, t) d t .
$$

To simplify the notation we introduce $\phi_{1}(x)=T_{1}(x) g_{1}(x)$, and to obtain a differential equation for $\phi_{1}(x)$ we differentiate equation (3.6) twice with respect to $x$ and integrate equation (3.1) with respect to $t$ from zero to infinity. Combining these two results gives

$$
D \frac{d^{2} \phi_{1}(x)}{d x^{2}}-k_{1} \phi_{1}(x)=-g_{1}(x)
$$


The boundary conditions for equation (3.7) require that $\phi_{1}(0)=0$ and equation (3.6) implies that $\phi_{1}(x)$ approaches zero as $x \rightarrow \infty$. We solve (3.7) for $\phi_{1}(x)$, and then rearrange for $T_{1}(x)$, which can be written as

$$
T_{1}(x)=\frac{x \alpha_{1}}{2 k_{1}} .
$$

To calculate the VAT for $C_{1}(x, t)$ we start with

$$
\begin{aligned}
V_{1}(x) & =\int_{0}^{\infty}\left(t-T_{1}(x)\right)^{2} f_{1}(t ; x) d t \\
& =\frac{1}{g_{1}(x)} \int_{0}^{\infty}\left(t-T_{1}(x)\right)^{2} \frac{\partial}{\partial t}\left(C_{1}(x, t)-C_{1 \infty}(x)\right) d t .
\end{aligned}
$$

Expanding the square term in equation (3.9), we rewrite two of the three integrals on the right in terms of $T_{1}(x)$, which gives

$$
V_{1}(x)+T_{1}(x)^{2}=\frac{2}{g_{1}(x)} \int_{0}^{\infty} t \frac{\partial}{\partial t}\left(C_{1 \infty}(x)-C_{1}(x, t)\right) d t .
$$

To simplify the notation we introduce $\psi_{1}(x)=g_{1}(x)\left(V_{1}(x)+T_{1}(x)^{2}\right)$. We obtain a differential equation for $\psi_{1}(x)$ by differentiating equation (3.10) twice with respect to $x$, multiplying equation (3.1) by $t$, and integrating that expression with respect to $t$ from zero to infinity. Combining these results gives

$$
D \frac{d^{2} \psi_{1}(x)}{d x^{2}}-k_{1} \psi_{1}(x)=-2 \phi_{1}(x) .
$$

The boundary conditions for equation (3.11) require that $\psi_{1}(0)=0$ and equation (3.10) implies that $\psi_{1}(x)$ approaches zero as $x \rightarrow \infty$. We solve (3.11) for $\psi_{1}(x)$ and then rearrange for the VAT, which can be written as

$$
V_{1}(x)=\frac{x \alpha_{1}}{4 k_{1}^{2}} .
$$

To derive expressions for the MAT and VAT for $C_{2}(x, t)$ we rewrite equation (3.2) as

$$
\frac{\partial S}{\partial t}=D \frac{\partial^{2} S}{\partial x^{2}}-k_{2} C_{2}
$$

where $S(x, t)=C_{1}(x, t)+C_{2}(x, t)$. For our initial conditions and boundary conditions the steady state can be written as

$$
S_{\infty}(x)=\frac{1}{k_{2}-k_{1}}\left[k_{2} \exp \left(-x \alpha_{1}\right)-k_{1} \exp \left(-x \alpha_{2}\right)\right]
$$

where $k_{2} \neq k_{1}$. Following our procedure for $T_{1}(x)$ and $V_{1}(x)$, we identify

$$
F_{2}(t ; x)=1-\frac{S(x, t)-S_{\infty}(x)}{S(x, 0)-S_{\infty}(x)}
$$


as a cumulative distribution function, and the associated probability density function is given by

$$
f_{2}(t ; x)=-\frac{\partial}{\partial t}\left[\frac{S(x, t)-S_{\infty}(x)}{S(x, 0)-S_{\infty}(x)}\right] .
$$

With these definitions we can write the MAT as

$$
T_{2}(x)=\int_{0}^{\infty} t f_{2}(t ; x) d t=\frac{1}{g_{2}(x)} \int_{0}^{\infty} t \frac{\partial}{\partial t}\left(S(x, t)-S_{\infty}(x)\right) d t .
$$

To simplify the notation we introduce $\phi_{2}(x)=T_{2}(x) g_{2}(x)$, and to obtain a differential equation for $\phi_{2}(x)$ we differentiate equation (3.14) twice with respect to $x$ and integrate equation (3.13) with respect to $t$ from zero to infinity. Combining these two results gives

$$
D \frac{d^{2} \phi_{2}(x)}{d x^{2}}-k_{2} \phi_{2}(x)=-g_{2}(x)-k_{2} \phi_{1}(x) \text {. }
$$

The boundary conditions for equation (3.15) require that $\phi_{2}(0)=0$, and equation (3.14) implies that $\phi_{2}(x)$ approaches zero as $x \rightarrow \infty$. Using Maple we solve for $\phi_{2}(x)$ and, using this result, we find an expression for $T_{2}(x)$ which can be written as

$$
T_{2}(x)=\frac{\exp \left(-\alpha_{2} x\right)\left[k_{1} x \sqrt{k_{1} k_{2}}-2 k_{2} \sqrt{k_{1} D}\right]+\exp \left(-\alpha_{1} x\right)\left[2 k_{2} \sqrt{k_{2} D}-k_{2}^{2} x\right]}{2 k_{1} k_{2} \sqrt{k_{1} D}\left[\exp \left(-\alpha_{2} x\right)-\exp \left(-\alpha_{1} x\right)\right]} .
$$

To calculate the VAT we start with

$$
\begin{aligned}
V_{2}(x) & =\int_{0}^{\infty}\left(t-T_{2}(x)\right)^{2} f_{2}(t ; x) d t \\
& =\frac{1}{g_{2}(x)} \int_{0}^{\infty}\left(t-T_{2}(x)\right)^{2} \frac{\partial}{\partial t}\left(S(x, t)-S_{\infty}(x)\right) d t .
\end{aligned}
$$

Expanding the square term in equation (3.16), we rewrite two of the three integrals on the right in terms of $T_{2}(x)$, which gives

$$
V_{2}(x)+T_{2}(x)^{2}=\frac{2}{g_{2}(x)} \int_{0}^{\infty} t \frac{\partial}{\partial t}\left(S_{\infty}(x)-S(x, t)\right) d t .
$$

To simplify the notation we introduce $\psi_{2}(x)=g_{2}(x)\left(V_{2}(x)+T_{2}(x)^{2}\right)$. We obtain a differential equation for $\psi_{2}(x)$ by differentiating equation (3.17) twice with respect to $x$, after multiplying equation (3.13) by $t$ we integrate that expression with respect to $t$ from zero to infinity, and combining these two expressions we arrive at

$$
D \frac{d^{2} \psi_{2}(x)}{d x^{2}}-k_{2} \psi_{2}(x)=-k_{2} \psi_{1}(x)-\phi_{2}(x) .
$$

The boundary conditions for equation (3.18) require that $\psi_{2}(0)=0$ and equation (3.16) implies that $\psi_{2}(x)$ approaches zero as $x \rightarrow \infty$. Using Maple we solve for $\psi_{2}(x)$ and then 
rearrange $V_{2}(x)$, which can be written as

$$
\begin{aligned}
V_{2}(x)= & \frac{1}{k_{1}^{3 / 2}} k_{2}^{2} D^{3 / 2}\left(k_{1}-k_{2}\right)^{2}\left[\exp \left(-\alpha_{2} x\right)-\exp \left(-\alpha_{1} x\right)\right] \\
& \times\left\{\operatorname { e x p } ( - \alpha _ { 2 } x ) k _ { 1 } ^ { 3 / 2 } \left[k_{1}^{2} k_{2} \sqrt{D} x^{2}-k_{1} k_{2}^{2} \sqrt{D} x^{2}+k_{1}^{2} \sqrt{k_{2}} D x-5 k_{1} k_{2}^{3 / 2} D x\right.\right. \\
& \left.+4 k_{2}^{5 / 2} x+8 k_{2}^{2} D^{3 / 2}\right]-\exp \left(-\alpha_{1} x\right) k_{2}^{2}\left[8\left(k_{1} D\right)^{3 / 2}+k_{2} k_{1}^{3 / 2} \sqrt{D} x^{2}\right. \\
& \left.\left.+k_{1} k_{2} D x-k_{2}^{2} \sqrt{k_{1} D} x^{2}-k_{2}^{2} D x\right]\right\} .
\end{aligned}
$$

3.2. Results Results in Figure 2(a)-(b) show a numerical solution of equations (3.1)-(3.2) for $C_{1}(x, 0)=C_{2}(x, 0)=0, C_{1}(0, t)=1$ and $C_{2}(0, t)=0$. Although we are interested in solving equations (3.1)-(3.2) on the semi-infinite domain $0 \leq x<$ $\infty$, our numerical calculations were performed on a sufficiently long finite domain $0 \leq x \leq L$, with homogeneous Dirichlet boundary conditions at $x=L$, to ensure that our choice of $L$ had no impact on the evolution of the numerical solutions. All results in Figure 2 correspond to $L=10000$ (we checked that our results were insensitive to this choice by recomputing our results with $L=100000$, which showed that the two sets of numerical results were indistinguishable). The steady-state solution of (3.1)-(3.2), given by (3.3)-(3.4), is superimposed on the transient solutions in Figure 2(a)-(b). Comparing the transient and steady-state solutions indicates that the first component, $C_{1}(x, t)$, appears to approach steady state faster than the second component, $C_{2}(x, t)$, since we see that the $C_{1}(x, 500)$ profile is practically indistinguishable from the corresponding steady state, $C_{1 \infty}(x)$, whereas the $C_{2}(x, 500)$ profile is visually distinguishable from the corresponding steady state, $C_{2 \infty}(x)$.

To illustrate our MAT and VAT results for equations (3.1)-(3.2), numerical results illustrating the temporal evolution of $C_{1}(x, t)$ and $C_{2}(x, t)$, at a fixed location $x=200$, are shown in Figure 2(c)-(d), respectively. The profile in Figure 2(c) confirms that $C_{1}(200, t)$ asymptotes to the corresponding steady state as $t \rightarrow \infty$ by plotting $C_{1 \infty}(200)-C_{1}(200, t)$, which decays to zero in the long-time limit. Our results, given by equations (3.8) and (3.12), indicate that $T_{1}(200)=447.21$ days and $V_{1}(200)=$ $211.47^{2}$ days $^{2}$ for this particular application. These values, illustrated in Figure 2(c), confirm that the MAT and VAT provide a useful indication of the critical time interval for this species to evolve to the corresponding steady state. Similarly, our analysis shows that $T_{2}(200)=554.93$ days and $V_{2}(200)=347.03^{2}$ days ${ }^{2}$, which, in comparison with the numerical profile in Figure 2(d), provides a useful indication of the critical time interval for the evolution of the $C_{2}(x, t)$ profile.

\section{Discussion and conclusions}

In 1991, McNabb introduced the concept of MAT as a physically reasonable and mathematically convenient finite measure of the time required for a diffusive process to effectively reach steady state [21, 22]. McNabb's definition of MAT is physically reasonable since it gives a finite measure of the time that corresponds to the mean time 

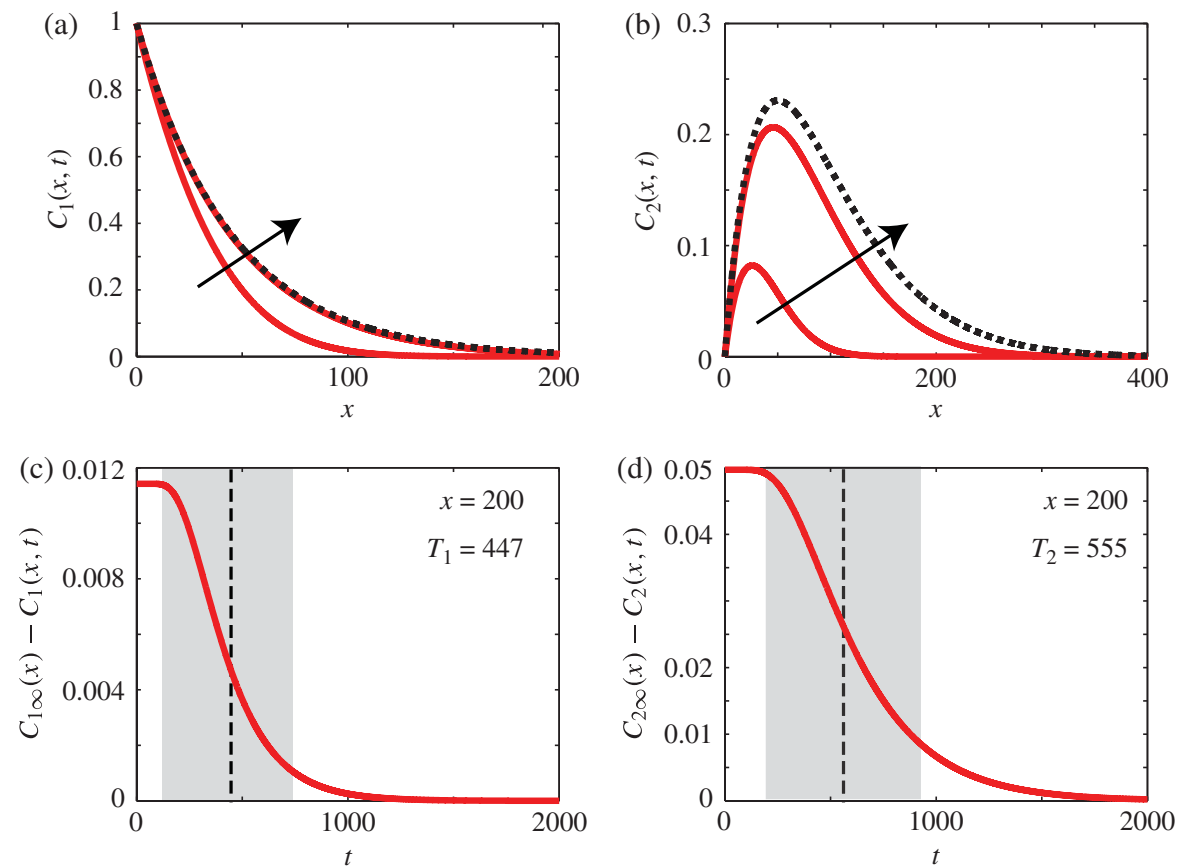

FIgure 2. Profiles in (a)-(b) show numerical solutions of (3.1)-(3.2) with $C_{1}(0, t)=0, C_{2}(0, t)=0$, $D=10, k_{1}=0.005$ and $k_{2}=0.003$. Numerical solutions were obtained on the truncated domain $0 \leq$ $x \leq 10000$, using a central difference approximation with a uniform spatial discretization $\delta x=0.1$ and implicit Euler temporal integration with time step $\delta t=0.1[23,24]$. Transient solutions are shown at $t=100$ and $t=500$ (solid lines) with the arrow indicating the direction of increasing $t$. The corresponding steady state solutions, given by (3.3)-(3.4), are shown in black (dotted). Profiles in (c)-(d) show how $C_{1 \infty}(x)-C_{1}(x, t)$ and $C_{2 \infty}(x)-C_{2}(x, t)$ decay to zero as $t \rightarrow \infty$, at the location $x=200$. The MATs, $T_{1}(200)=447.21$ and $T_{2}(200)=554.93$ days, are superimposed on the numerical results in (c) $-(\mathrm{d})$ using a vertical line (dashed). We also indicate, by shading, the critical time interval for each species, $\left[T_{i}-\sqrt{V_{i}}, T_{i}+\sqrt{V_{i}}\right]$. Here $V_{1}(200)=211.47^{2}$ and $V_{2}(200)=347.03^{2}$ days $^{2}$.

required for the transient solution of the process to asymptote to the corresponding steady state. Other definitions of critical time, such as the amount of time required for a diffusive process to reach within $1 \%$ of steady state, are less satisfactory since the choice of threshold is arbitrary and such estimates of the critical time depend upon the threshold value $[21,22]$. McNabb's definition of MAT is mathematically convenient since it is possible to determine the MAT without solving the underlying model for the transient solution [17, 21, 22]. Recently, Berezhkovskii and co-workers introduced a critical time, called the LAT, as a measure of the time required for morphogen gradients to form during embryonic development [2-4, 11, 15]. Our recent work showed that Berezhkovskii's definition of LAT is equivalent to McNabb's definition of MAT [8]. Furthermore, we showed that the MAT corresponds to the mean value of a certain probability density function and that it is also straightforward to evaluate the higher moments of this probability density function, such as the VAT [9]. 
All previous analyses of MAT, and associated concepts, have studied ODE and PDE models with a single dependent variable. Motivated by the fact that many practical models are based on coupled processes, we applied McNabb's definition of MAT to coupled models. Our work shows that a change of variables is required so that we can define an appropriate cumulative distribution function and probability density function from which we can evaluate the mean, variance and other moments. We have presented results for a system of coupled ODEs with applications that include the study of coupled chemical and biochemical decay processes [29]. Our analysis shows that it is straightforward to give a general expression for the MAT and VAT for such a system with an arbitrary number of species. This analysis is relatively straightforward since the concentration of all species approaches zero in the long-time limit.

We have also extended our results to the study of coupled PDEs. Instead of presenting general results for a system with an arbitrary number of species, we have outlined a general framework and developed illustrative results for a system of coupled PDEs with two species. Specific results were presented for a particular set of initial conditions and boundary conditions used previously to study coupled chemical transport and decay [5, 19]. Although our results correspond to a particular application of the two-species coupled PDE model, our framework for deriving the boundary value problems governing the MAT and VAT for each species can be extended to other situations, such as models that include advective transport, models with more than two species, or models with nonzero initial conditions.

The techniques that we used to compute and implement the MAT and VAT results could be modified in future applications of this work. We obtained our results for the MAT and VAT of the second component of the coupled PDE model using symbolic computation. For more detailed problems, such as those involving advective transport, more than two species, or more complicated boundary conditions and initial conditions, we expect that the solutions of the relevant boundary value problems for the MAT and VAT would be more conveniently sought using numerical techniques. Here, we used the VAT and MAT to define a critical time interval as the mean plus or minus one standard deviation. It is also possible to consider a different definition of critical time interval, such as the mean plus or minus two standard deviations, and this choice might depend on the particular details of the application.

The work outlined here can be applied to study other models, such as two- and threedimensional diffusive processes. For such linear models the techniques developed here for one-dimensional linear diffusive processes are directly applicable except that the equations governing the MAT and VAT will be two- and three-dimensional boundary value problems that could be solved using standard techniques on separable domains. Other problems, such as finding the MAT and VAT of nonlinear processes, including processes governed by nonlinear diffusion $[10,26]$ and nonlinear reaction-diffusion equations [25], are far more challenging. The MAT and VAT for such processes can be analysed approximately, using techniques such as developing upper and lower bounds for the MAT [11] or implementing approximate relationships between the time evolution of the dependent variable and the MAT [8]. As far as we are aware, there 
has been no progress made towards analysing the MAT and VAT of coupled nonlinear processes, and we suggest that this will be a fruitful area for future research.

\section{Acknowledgements}

M. J. Simpson and R. E. Baker acknowledge support from the Australian Research Council Discovery Project DP120100551 and the 2011 International Exchange Scheme funded by the Royal Society.

\section{Appendix A. Integral evaluations}

To demonstrate how to evaluate the integral expressions in equation (2.8) we consider the system given by equations (2.1)-(2.4) for $N=4$ species. The transformed variable, $S_{n}(t)=\sum_{i=1}^{n} C_{i}(t)$, decays to zero exponentially fast as $t \rightarrow \infty$. Therefore, integration by parts gives us

$$
\begin{aligned}
& \int_{0}^{\infty} t \frac{d S_{1}}{d t} d t=-\int_{0}^{\infty} C_{1}(t) d t \\
& \int_{0}^{\infty} t \frac{d S_{2}}{d t} d t=-\int_{0}^{\infty} C_{1}(t)+C_{2}(t) d t \\
& \int_{0}^{\infty} t \frac{d S_{3}}{d t} d t=-\int_{0}^{\infty} C_{1}(t)+C_{2}(t)+C_{3}(t) d t \\
& \int_{0}^{\infty} t \frac{d S_{4}}{d t} d t=-\int_{0}^{\infty} C_{1}(t)+C_{2}(t)+C_{3}(t)+C_{4}(t) d t
\end{aligned}
$$

Using (2.1)-(2.4) we rewrite, and evaluate, the integrals on the right-hand side of (A.1)-(A.4) to give

$$
\begin{aligned}
\int_{0}^{\infty} C_{1}(t) d t & =\frac{C_{1}(0)}{k_{1}}, \\
\int_{0}^{\infty} C_{1}(t)+C_{2}(t) d t & =\frac{C_{1}(0)}{k_{1}}+\frac{C_{1}(0)+C_{2}(0)}{k_{2}}, \\
\int_{0}^{\infty} C_{1}(t)+C_{2}(t)+C_{3}(t) d t & =\frac{C_{1}(0)}{k_{1}}+\frac{C_{1}(0)+C_{2}(0)}{k_{2}} \\
& +\frac{C_{1}(0)+C_{2}(0)+C_{3}(0)}{k_{3}}, \\
\int_{0}^{\infty} C_{1}(t)+C_{2}(t)+C_{3}(t)+C_{4}(t) d t=\frac{C_{1}(0)}{k_{1}}+\frac{C_{1}(0)+C_{2}(0)}{k_{2}} & +\frac{C_{1}(0)+C_{2}(0)+C_{3}(0)}{k_{3}} \\
+ & \frac{C_{1}(0)+C_{2}(0)+C_{3}(0)+C_{4}(0)}{k_{4}} .
\end{aligned}
$$

The expressions in equations (A.5)-(A.8) generalize to the first expression in equation (2.8) for arbitrary $N>1$. 
Again, noting that $S_{n}(t)$ decays to zero exponentially fast as $t \rightarrow \infty$, we use integration by parts, together with equations (2.1)-(2.4), to give

$$
\begin{aligned}
\int_{0}^{\infty} t^{2} \frac{d S_{1}}{d t} d t & =\frac{2}{k_{1}} \int_{0}^{\infty} t \frac{d S_{1}}{d t} d t \\
\int_{0}^{\infty} t^{2} \frac{d S_{2}}{d t} d t & =\frac{2}{k_{1}} \int_{0}^{\infty} t \frac{d S_{1}}{d t} d t+\frac{2}{k_{2}} \int_{0}^{\infty} t \frac{d S_{2}}{d t} d t \\
\int_{0}^{\infty} t^{2} \frac{d S_{3}}{d t} d t= & \frac{2}{k_{1}} \int_{0}^{\infty} t \frac{d S_{1}}{d t} d t+\frac{2}{k_{2}} \int_{0}^{\infty} t \frac{d S_{2}}{d t} d t+\frac{2}{k_{3}} \int_{0}^{\infty} t \frac{d S_{3}}{d t} d t \\
\int_{0}^{\infty} t^{2} \frac{d S_{4}}{d t} d t= & \frac{2}{k_{1}} \int_{0}^{\infty} t \frac{d S_{1}}{d t} d t+\frac{2}{k_{2}} \int_{0}^{\infty} t \frac{d S_{2}}{d t} d t+\frac{2}{k_{3}} \int_{0}^{\infty} t \frac{d S_{3}}{d t} d t \\
& +\frac{2}{k_{4}} \int_{0}^{\infty} t \frac{d S_{4}}{d t} d t
\end{aligned}
$$

The integrals on the right-hand sides of equations (A.9)-(A.12) can be evaluated using the results in equations (A.5)-(A.8). Together, these expressions generalize to the second expression in equation (2.8) for arbitrary $N>1$.

\section{References}

[1] G. I. Barenblatt, Scaling (Cambridge University Press, Cambridge, 2003).

[2] A. M. Berezhkovskii, C. Sample and S. Y. Shvartsman, "How long does it take to establish a morphogen gradient?" Biophys. J. 99 (2010) L59-L61; doi:10.1016/j.bpj.2010.07.045.

[3] A. M. Berezhkovskii, C. Sample and S. Y. Shvartsman, "Formation of morphogen gradients: Local accumulation time", Phys. Rev. E 83 (2011) 051906; doi:10.1103/PhysRevE.83.051906.

[4] A. M. Berezhkovskii and S. Y. Shvartsman, "Physical interpretation of mean local accumulation time of morphogen gradient formation", J. Chem. Phys. 135 (2011) 154115; doi:10.1063/1.3654159.

[5] C. M. Cho, "Convective transport of ammonium with nitrification in soil", Canad. J. Soil Sci. 51 (1970) 339-350; doi:10.4141/cjss71-047.

[6] T. P. Clement, Y. Sun, B. S. Hooker and J. N. Petersen, "Modelling multispecies reactive transport in ground water", Ground Water Modeling and Remediation 18 (1998) 79-92; doi:10.1111/j.1745-6592.1998.tb00618.x.

[7] P. K. Denman, D. L. S. McElwain, D. G. Harkin and Z. Upton, "Mathematical modelling of aerosolised skin grafts incorporating keratinocyte clonal subtypes", Bull. Math. Biol. 69 (2007) 157-179; doi:10.1007/S11538-006-9082-z.

[8] A. J. Ellery, M. J. Simpson, S. W. McCue and R. E. Baker, "Critical timescales for advectiondiffusion-reaction processes", Phys. Rev. E 85 (2012) 041135; doi:10.1103/PhysRevE.85.041135.

[9] A. J. Ellery, M. J. Simpson, S. W. McCue and R. E. Baker, "Moments of action provide insight into critical times for advection-diffusion-reaction processes", Phys. Rev. E 86 (2012) 031136; doi:10.1103/PhysRevE.86.031136.

[10] A. E. Fernando, K. A. Landman and M. J. Simpson, "Nonlinear diffusion and exclusion processes with contact interactions", Phys. Rev. E 81 (2010) 011903; doi:10.1103/PhysRevE.81.011903.

[11] P. V. Gordon, C. Sample, A. M. Berezhkovskii, C. V. Muratov and S. Y. Shvartsman, "Local kinetics of morphogen gradients", Proc. Natl Acad. Sci. 108 (2011) 6157-6162; doi:10.1073/pnas.1019245108.

[12] R. I. Hickson, S. I. Barry and G. N. Mercer, "Critical times in multilayer diffusion. Part 1: Exact solutions", Int. J. Heat Mass Transfer 52 (2011) 5776-5783;

doi:10.1016/j.ijheatmasstransfer.2009.08.013. 
[13] R. I. Hickson, S. I. Barry and G. N. Mercer, "Critical times in multilayer diffusion. Part 2: Approximate solutions”, Int. J. Heat Mass Transfer 52 (2011) 5784-5791;

doi:10.1016/j.ijheatmasstransfer.2009.08.012.

[14] R. I. Hickson, S. I. Barry, H. S. Sidhu and G. N. Mercer, "Critical times in single-layer reaction diffusion”, Int. J. Heat Mass Transfer 54 (2011) 2642-2650; doi:10.1016/j.ijheatmasstransfer.2011.01.019.

[15] A. B. Kolomeisky, "Formation of a morphogen gradient: acceleration by degradation", J. Phys. Chem. Lett. 2 (2011) 1502-1505; doi:10.1021/jz2004914.

[16] K. A. Landman, A. Q. Cai and B. D. Hughes, "Travelling waves of attached and detached cells in a wound-healing cell migration assay”, Bull. Math. Biol. 69 (2007) 2119-2138; doi:10.1007/S11538-007-9206-0.

[17] K. A. Landman and M. J. McGuinness, "Mean action time for diffusive processes", J. Appl. Math. Decision Sci. 4 (2000) 125-141; doi:10.1155/S1173912600000092.

[18] K. A. Landman and L. R. White, "Predicting filtration time and maximizing throughput in a pressure filter", AIChE J. 43 (1997) 3147-3160; doi:10.1002/aic.690431204.

[19] M. Lunn, R. J. Lunn and R. Mackay, "Determining analytic solutions of multiple species contaminant transport, with sorption and decay", J. Hydrol. 180 (1996) 195-210; doi:10.1016/0022-1694(95)02891-9.

[20] J. H. Montgomery, Groundwater chemicals desk reference, 4th edn. (CRC Taylor and Francis, Boca Raton, FL, 2007).

[21] A. McNabb, "Mean action times, time lags, and mean first passage times for some diffusion problems", Math. Comput. Modell. 18 (1993) 123-129; doi:10.1016/0895-7177(93)90221-J.

[22] A. McNabb and G. C. Wake, "Heat conduction and finite measures for transition times between steady states", IMA J. Appl. Math. 47 (1991) 193-206; doi:10.1093/imamat/47.2.193.

[23] M. J. Simpson and K. A. Landman, "Analysis of split operator methods applied to reactive transport with Monod kinetics", Adv. Water Resour. 30 (2007) 2026-2033; doi:10.1016/j.advwatres.2007.04.005.

[24] M. J. Simpson, K. A. Landman and T. P. Clement, "Assessment of a nontraditional operator split algorithm for simulation of reactive transport", Math. Comput. Simul. 70 (2005) 44-60; doi:10.1016/j.matcom.2005.03.019.

[25] M. J. Simpson, K. A. Landman and B. D. Hughes, "Cell invasion with proliferation mechanisms motivated by time-lapse data", Phys. A 389 (2010) 3779-3790; doi:10.1016/j.physa.2010.05.020.

[26] M. J. Simpson, C. Towne, D. L. S. McElwain and Z. Upton, "Migration of breast cancer cells: Understanding the roles of volume exclusion and cell-to-cell adhesion", Phys. Rev. E 82 (2010) 041901; doi:10.1103/PhysRevE.82.041901.

[27] M. Th. van Genuchten, "Convective-dispersive transport of solutes involved in sequential firstorder decay reactions", Comput. Geosci. 11 (1985) 129-147; doi:10.1016/0098-3004(85)90003-2.

[28] T. M. Vogel and P. L. McCarty, "Biotransformation of tetrachloroethylene to trichloroethylene, dichloroethylene, vinyl chloride, and carbon dioxide under methanogenic conditions", Appl. Environ. Microbiol. 49 (1985) 1080-1083; http://www.ncbi.nlm.nih.gov/pmc/articles/PMC238509/.

[29] C. Z. Zheng and G. D. Bennett, Applied contaminant transport modeling, 2nd edn. (John Wiley, New York, 2002). 